\title{
Atypical femoral fractures
}

\author{
Sandro Giannini \\ Eugenio Chiarello \\ Giuseppe Tedesco \\ Matteo Cadossi \\ Deianira Luciani \\ Antonio Mazzotti \\ Davide Maria Donati
}

$1^{\text {st }}$ Orthopaedic and Traumatologic Clinic, Rizzoli Orthopaedic Institute, University of Bologna, Bologna, Italy

Address for correspondence:

Eugenio Chiarello, MD

$1^{\text {st }}$ Orthopaedic and Traumatologic Clinic

Rizzoli Orthopaedic Institute

University of Bologna

Via G.C. Pupilli, 1

40134 Bologna, Italy

Phone/Fax: +390516366581

E-mail: eugenio.chiarello@yahoo.it

\section{Summary}

Bisphosphonates (BPs) represent the most widely used therapy for osteoporosis. Recently, a relationship between long-term treatment with BPs and a subset of atypical femoral fractures (AFFs) from below the lesser trochanter to the sovracondilar line has been described. Many etiopathogenetic theories have been invoked to explain AFFs: reduced bone turnover and increased osteoblast bone apposition with accumulation of microdamage and decreased bone toughness with subsequent increased risk of micro-cracks and duration fractures, collagen fiber cross-linking and vascularization impairment.

Based on published studies, a task force of the American Society for Bone and Mineral Research has redacted the diagnostic criteria of AFFs by classifying them according to their major and minor criteria.

The treatment for displaced AFFs is osteosynthesis, but there is a lack of evidence for undisplaced AFFs and the duration of fracture treatment. BPs have a proven efficacy in osteoporotic fracture reduction as well as in the treatment of other bone diseases caused by the downregulation of osteoclast activity. BPs have an excellent benefit-to-risk ratio; however, minor adverse events, such as AFFs, occur in a variable percentage of patients treated over a long period of time.

KEY WORDS: atypical femoral fractures; bisphosphonates; surgical treatment.

\section{Introduction}

Osteoporosis is a chronic disease whose incidence is increasing due to the aging of the population $(1,2)$. This pathology requires a long-term therapy to prevent fragility fractures. Currently there are several drug classes for the treatment of osteoporosis: estrogen, selective estrogen receptor modulators (SERMs) (such as raloxifene and bazedoxifene), antiresorptive drugs [such as bisphosphonates (BPs)], anabolic drugs [such as recombinant human parathormon (rh-PTH)], dual action drug (such as strontium ranelate) and monoclonal antibodies (such as Denosumab) (3-5). Nowadays, BPs represent the most widely used therapy for osteoporosis and other osteoclast-related bone diseases, such as Paget and bone metastases. BPs are also indicated in the therapy for Osteogenesis Imperfecta (6).

BPs are potent inhibitors of osteoclast-mediated bone resorption, with a demonstrated effectiveness in reducing the incidence of vertebral, femoral and other fragility fractures in both women and men (7).

The efficacy of BPs varies among patients. It is estimated that up to $15 \%$ of patients fail to respond to BPs therapy (8).

Moreover, some patients undergoing intravenous BPs treatment have experienced adverse events, such as atrial fibrillation, acute phase response, renal insufficiency and osteonecrosis of the jaw.

Recently, a relationship between long-term treatment with BPs and a subset of atypical femoral fractures (AFFs) from below the lesser trochanter to the sovracondilar line (mostly at the subtronchateric region) has been described. The first report was published by Odvina (2005) who described 9 cases in women having severely suppressed bone turnover who sustained AFFs and had been taking BPs for at least 1 year (9). Subsequently, several clinical cases and case reviews reported an association between low energy trauma, subtronchateric or femoral shaft fractures and patients who had been treated with long-term BPs therapy.

Kim et al. reported an analysis based on data from the United States showing how rare AFFs are: of 33,851 patients treated with BPs, only 104 sustained an AFF, with an estimated occurrence of 1.46 per 1,000 treated patients per year (10).

In a cohort study using national healthcare data of the U.S., Abrahamsen et al. compared 39,567 patients taking alendronate from 1996 to 2005 with 158,268 untreated controls, reporting that subtrochanteric and diaphyseal fractures occurred at a rate of 13 per 10,000 patient/year in untreated women and 31 per 10,000 patient/year in women receiving alendronate (11).

Subtronchateric or femoral shaft fractures are rare $(10-30 \%$ of all hip/femur fractures) and many of them (about $75 \%$ ) are usually associated with a high energy trauma. Subtronchateric fractures account for approximately $3 \%$ of all femoral fractures in the elderly, but the morbidity and mortality are similar to hip fractures.

\section{Etiopathogenesis}

The etiopathogenesis of AFFs is still unknown; studies of the effects of BPs on bone metabolism suggest involvement of several biological mechanisms.

BPs have a strong affinity for mineralized tissue; they cause a chemical effect by binding strongly to calcium crystals and then inducing a cellular effect on the osteoclasts during bone re- 
sorption. BPs have a long half-life, thus affecting osteoclast activity for several months after the end of therapy. By reducing bone turnover, BPs treatment leads to increased bone microdamage and decreased bone toughness with a subsequently higher risk of micro-cracks and duration fractures (12).

These findings were clearly demonstrated by Mashiba and Komatsubara in animals models showing that suppressed bone turnover results in the accumulation of micro-damage and the reduction of bone toughness without a reduction in strength (13-15).

Human bone biopsies in patients who have taken BPs have shown conflicting results; an analysis of several studies with a total of 57 biopsies performed on the iliac crest or at the fracture site showed low bone turnover in the majority of patients but not in all of them. However, caution should be used when interpreting a biopsy at the fracture site because the fracture itself speeds up the remodelling processes (16).

Other theories of etiopathogenesis have hypothesized the involvement of collagen fibre cross-linking; BPs lead to increased mature collagen shaping, and greater bone stiffness and strength $(17,18)$.

Other authors have found that BPs reduce bone turnover, thus increasing bone mineralization and leaving the mineral part unmodified. This mineralization enhancement produces increased bone stiffness and strength $(19,20)$.

Moreover, Donnelly et al. compared the bones of 20 women taking BPs (with an average time of 7 years of treatment) with 20 naïve osteoporotic women. This study showed that the BP patients had more uniform bone tissue, especially in the cortical bone (21).

Another hypothesis for explaining the etiopathogenesis of AFFs is the vascularization impairment due to the BPs intake. Wood observed that BPs have an anti-angiogenic action (22); nevertheless, this finding was not confirmed by an animal study with clodronates (23). Other authors have found a vascularization reduction of up to $40 \%$ in mice treated with BPs and have hypothesized that this reduction was due to osteoclastic activity inhibition (24).

\section{Diagnosis}

Approximately $70 \%$ of patients with a confirmed stress fracture report prodromal pain before diagnosis (16). Although AFFs are uncommon, they should always be suspected in elderly patients with chronic pain. The time between the onset of the pain and the diagnosis of an AFF varies from one week to 2 years (25).

When an AFF is clinically suspected, an X-ray should be carried out in both the antero-posterior and the lateral views. The data from case reports and case series show that BP-related AFFs present specific radiographic features (26).

Based on published studies, a task force of the American Society for Bone and Mineral Research (ASBMR) has redacted the diagnostic criteria of AFFs by classifying them according to their major and minor criteria (16) (Table 1). For the diagnosis of an atypical fracture, all major criteria are required; minor criteria could be either present or not. The X-ray characteristics of AFFs are listed in Table 2.

Unlike stress fractures, which usually involve the medial cortex of the proximal third of the femoral diaphysis, in AFFs, the fracture line starts from the lateral cortex (Figure $1 \mathrm{~A}, \mathrm{~B}$ ). Moreover, stress fractures are commonly observed in athletes while AFFs are typical of elderly patients.

When groin or thigh pain is present with negative X-rays, it is necessary to perform a second level exam, such as bone scintigraphy, MRI or a CT scan.

Bone scintigraphy may show focal increased uptake at the lateral cortex; this feature should be unilateral or bilateral. MRI has greater sensitivity and specificity than scintigraphy. Similarly, a CT scan with thin section and multiplanar images makes this technique useful for identifying bony changes (16).

Table 1 - Diagnostic criteria for atypical femoral fracture (Task force for the American Society for Bone and Mineral Research) (16). Diagnosis requires the presence of all the major criteria. None of the minor criteria is required, but they could may be present concomitantly with major criteria.

\begin{tabular}{|c|c|c|}
\hline Major criteria & Minor criteria & Exclusion criteria \\
\hline $\begin{array}{l}\text { Proximal fracture line under the lesser } \\
\text { trochanter and distal fracture line above } \\
\text { the femoral condyles }\end{array}$ & $\begin{array}{l}\text { Periosteal reaction along } \\
\text { the lateral cortex }\end{array}$ & Femoral neck fracture \\
\hline No trauma or low-energy trauma & Increased cortical thickness & $\begin{array}{l}\text { Intertrochanteric fracture with extension } \\
\text { to the subtrochanteric femur }\end{array}$ \\
\hline $\begin{array}{l}\text { Transverse or only slightly oblique } \\
\left.\text { fracture line (angle }<30^{\circ}\right)\end{array}$ & Prodrome pain in the groin or thigh & Periprosthetic fracture \\
\hline Non comminuted fracture & Bilateral fracture & $\begin{array}{l}\text { Pathological fracture related to a primary } \\
\text { bone tumor or bone metastasis }\end{array}$ \\
\hline $\begin{array}{l}\text { Complete fracture crossing from one cortex } \\
\text { to the other, with or without a medical cortical } \\
\text { beak or incomplete fracture (or fissure) } \\
\text { involving only the outer cortex }\end{array}$ & Delayed healing & - \\
\hline- & $\begin{array}{l}\text { Co-morbidities: rheumatoid arthritis, } \\
\text { vitamin D deficiency, hypophosphatasia }\end{array}$ & - \\
\hline- & $\begin{array}{l}\text { Concomitant treatments: bisphosphonates, } \\
\text { glucocorticoids, proton pump inhibitors }\end{array}$ & - \\
\hline
\end{tabular}


Table 2 - X-ray characteristic of complete or incomplete fracture.

Transverse fracture line

Cortical thickening (focal or diffuse), particularly in the lateral cortex

Focal cortical thickening may produce an appearance of cortical "beaking" or "flaring" adjacent to a transverse fracture line

In a complete fracture, an oblique component may be observed as a prominent medial spike

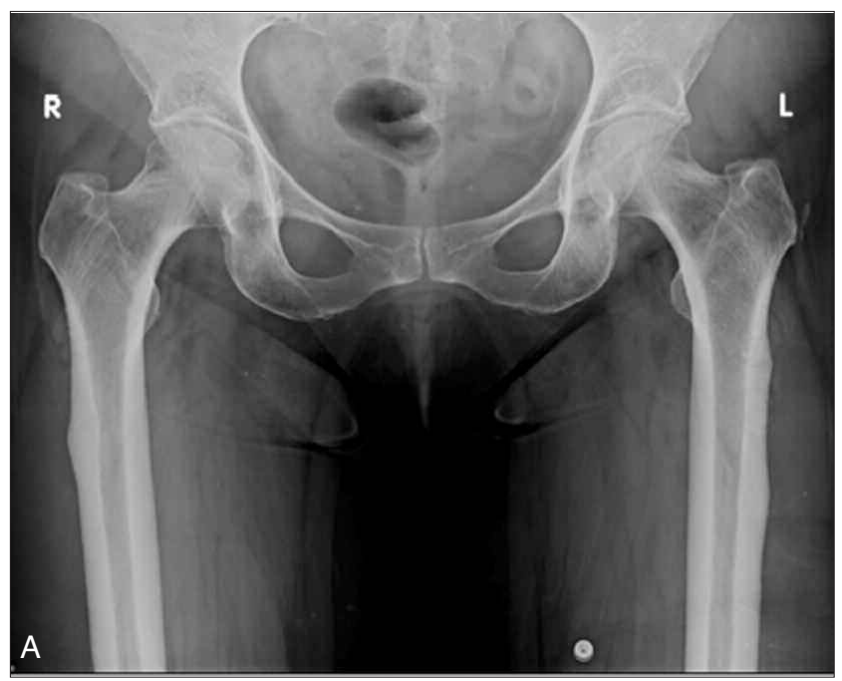

Figure 1A - A-P: X-ray of a 63 year old female under treatment with BPs for 5 years. The X-ray shows cortical thickening and beaking of the lateral cortex; a transverse fracture line is present.



Figure 1B - Lateral view which shows a duration fracture and femoral shaft narrowing.

\section{Treatment}

There is a lack of data in the literature regarding the appropriate surgical treatment. In the case of displaced fractures, the treatment should be reduction and surgical fixation. A contralateral femoral X-ray should always be carried out in order to eventually identify a contralateral AFF. If osteosynthesis is considered the treatment of choice for displaced AFFs, few data exist regarding the treatment of undisplaced AFFs.

Shane et al. have suggested that, when in the presence of an AFF, treatment should be guided by symptoms; asymptomatic patients should reduce their activity level, patients with slight groin or thigh pain should avoid weight bearing by using crutches. After 3 months of conservative treatment, when no symptomatic or radiological improvement occurs, a prophylactic fixation should be considered. In patients with moderate or severe pain and risk for fracture dislocation, prophylactic surgery should be performed (16).

For incomplete fractures intramedullary nail fixation seems to be easier; in the literature, no evidence exists as to which is the correct surgical technique for dislocated AFFs.

Despite the fact that Grady et al. (27) have reported two failures of AFFs healing after intramedullary nailing, other authors have reported good results, even if some additional procedures were required (28).

As BPs inhibit bone remodelling, in complete fractures, intramedullary nailing is preferred to open reduction and internal fixation with a plate and screws which leads to direct fracture healing where osteoclast activity is mandatory.

In the pharmacological management of patients affected by AFFs, the serum levels of calcium and vitamin D should always be assessed; when levels are low, standard supplements should be prescribed. Due to the possible etiopathogenesis of AFFs, all antiresorptive drugs should be suspended. Finally, in the literature, there are case reports in which the use of anabolic drugs, such as teriparatide, have improved fracture healing (29-32).

\section{Conclusion}

Bisphosphonates have a proven efficacy in the reduction of osteoporotic fractures as well as in the treatment of other bone diseases which benefit from a decrease in osteoclast activity. BPs have an excellent benefit-to-risk ratio; however, minor adverse events, such as AFFs, occur in a variable percentage of patients who have undergone long-term treatment.

In an analysis on 11,994 patients, Abrahamsen et al. showed that only $7 \%$ of patients with a subtrochanteric fracture of the femur had taken BPs (33).

Giusti et al. reported 141 atypical femoral fractures in women treated with BPs. In their study, $24 \%$ of the patients who sustained an AFF had been under treatment with BPs for less than 3 years and $75.9 \%$ had at least one major chronic disease: rheumatoid arthritis (10.3\%), asthma (14.9\%), diabetes (10.3\%) or chronic obstructive pulmonary disease (10\%). Moreover, $60 \%$ of the patients were using at least one drug other than BPs: estrogen (11.8\%), proton-pump inhibitors (38.9\%), more than $25 \%$ were on long-term oral glucocorticoid treatment and approximately $10 \%$ were using inhaled glucocorticoids (25).

The European Medicines Agency stated that AFFs are a class effect of BPs, but that the benefits arising from their use continue to outweigh its risks (34).

There is no rationale for withholding BPs therapy from patients with osteoporosis, although continued use of BPs beyond a treatment period of 3 to 5 years should be revaluated annually. Consideration should be given to stopping (at least temporarily) BPs therapy in patients who are reassessed to be at low or 
low-moderate risk (no incident fractures, T-score of 2.0 or higher, and no other major risk factors) after a 3 to 5 year therapeutic period $(35,36)$.

The possibility to predicting the risk of adverse drug reactions is essential for chronic bone disorders requiring long-term treatment. Moreover, various effective therapies are currently available and, therefore, selecting the best treatment option for each individual patient is a reality.

\section{References}

1. Komadina R. Hip, Osteoporosis: new paradigm. Eur J Trauma Emerg Surg 2008 April;34(2):163-170.

2. Lane NE. Epidemiology, etiology, and diagnosis of osteoporosis. Am J Obstet Gynecol 2006 feb;194(2 Suppl):S3-11.

3. Schuiling KD, Robinia K, Nye R. Osteoporosis update. J Midwifery Womens Health 2011 Nov- Dec;56(6):615-27.

4. Briot K, Cortet B, Thomas T, et al. 2012 update of French guidelines for the pharmacological treatment of postmenopausal osteoporosis. Joint Bone Spine 2012 may;79(3):304-13

5. Murad MH, Drake MT, Mullan RJ, et al. Clinical review. Comparative effectiveness of drug treatments to prevent fragility fractures: a systematic review and network meta-analysis. J Clin Endocrinol Metab 2012 jun;97(6):187180.

6. Cundy T. Recent advances in osteogenesis imperfecta. Calcif Tissue Int 2012 jun;90(6):439-49.

7. Khosla S, Bilezikian JP, Dempster DW, et al. Benefits and risks of bisphosphonate therapy for osteoporosis. J Clin Endocrinol Metab 2012 jul;97(7):2272-82.

8. Francis RM. Non-response to osteoporosis treatment. J Br Menopause Soc 2004 Jun;10(2):76-80.

9. Odvina CV, Zerwekh JE, Rao DS, et al. Severely suppressed bone turnover: a potential complication of alendronate therapy. J Clin Endocrinol Metab 2005 Mar;90(3):1294-301.

10. Kim SY, Schneeweiss S, Katz JN, et al. Oral bisphosphonates and risk of subtrochanteric or diaphyseal femur fractures in a population-based cohort. J Bone Miner Res 2011 May;26(5):993-1001.

11. Abrahamsen B, Eiken $P$, Eastell R. Cumulative alendronate dose and the long-term absolute risk of subtrochanteric and diaphyseal femur fractures: a register-based national cohort analysis. J Clin Endocrinol Metab 2010 Dec;95(12):5258-65.

12. Papapoulos SE. Bisphosphonates: How do they work? Best Pract Res Clin Endocrinol Metab 2008 Oct;22(5):831-47.

13. Li J, Mashiba T, Burr DB. Bisphosphonate treatment suppresses not only stochastic remodeling but also the targeted repair of microdamage. Calcif Tissue Int 2001Nov;69(5):281-6.

14. Komatsubara S, Mori S, Mashiba T, et al. Suppressed bone turnover by longterm bisphosphonate treatment accumulates microdamage but maintains intrinsic material properties in cortical bone of dog rib. J Bone Miner Res 2004 Jun;19(6):999-1005.

15. Mashiba T, Turner $\mathrm{CH}$, Hirano T, et al. Effects of suppressed bone turnover by bisphosphonates on microdamage accumulation and biomechanical properties in clinically relevant skeletal sites in beagles. Bone 2001May;28(5):524-31.

16. Shane E, Burr D, Ebeling PR, et al. Atypical subtrochanteric and diaphyseal femoral fractures: report of a task force of the American Society for Bone and Mineral Research. J Bone Miner Res 2010 Nov;25(11):2267-94.

17. Zioupos P, Currey JD, Hamer AJ. The rule of collagen in the declining mech- anism proprieties of aging human cortical bone. J Biomed Mather Res 1999 May;45(2):108-16.

18. Veashishth $\mathrm{D}$. Advanced glication end product and bone fractures. IBMS BoneKEy 2009;6: 268-278.

19. Boivin GY, Chavassieux PM, Santora AC, et al. Alendronate increase bone streinght by increasing the mean degree of mineralization of bone tissue in osteoporotic women. Bone 2000 Nov;27(5):687-94.

20. Roschger $\mathrm{P}$, Rinnerthaler $\mathrm{S}$, Yates $\mathrm{J}$, et al. Alendronate increase degree and uniformity of mineralization in cancellous bone an decrease the porosity in cortical bone of osteoporotic women. Bone 2001 Aug;29(2): 185-191.

21. Donnelly E, Meredith DS, Nguyen JT, et al. Reduced cortical bone compositional heterogeneity with bisphosphonate treatment in postmenopausal women with intertrochanteric and subtrochanteric fractures. J Bone Miner Res 2012 Mar;27(3):672-8.

22. Wood J, Bonjean K, Ruetz S, et al. Novel antiangiogenic effects of the bisphosphonate compound zoledronic acid. J Pharmacol Exp Ther 2002 Sep;302(3):1055-61.

23. Deckers MM, Van Beek ER, Van Der Pluijm G, et al. Dissotiation of angiogenesis and osteoclastogenesis during endochondral bone formation in neonatal mice. JBMR 2002 Jun;17(6):998-1007.

24. Fournier $\mathrm{P}$, Boissier $\mathrm{S}$, Filleur $\mathrm{S}$, et al. Bisphosphonates inhibit angiogenesis in vitro and testosterone-stimulated vascular regrowth in the ventral prostate in castrated rats. Cancer Res 2002 Nov 15;62(22):6538-44.

25. Giusti A, Hamdy NA, Papapoulos SE. Atypical fractures of the femur and bisphosphonate therapy: A systematic review of case/case series studies. Bone 2010 Aug;47(2):169-80.

26. Mohan PC, Howe TS, Koh JS, Png MA. Radiographic features of multifocal endosteal thickening of the femur in patients on long-term bisphosphonate therapy. Eur Radiol 2013 Jan;23(1):222-7.

27. Grady MK, Watson JT, Cannada LK. Treatment of femoral fracture nonunion after long-term bisphosphonate use. Orthopedics 2012 Jun;35(6):e991-5.

28. Weill YA, Rivkin G, Safran O, The outcome of surgically treated femur fractures associated with long-term bisphosphonate use. J Trauma 2011 Jul;71(1):186-90.

29. Fowler JR, Criner K, Craig MR. Prophylactic intramedullary fixation for bisphosphonate-related subtrochanteric stress fracture. Orthopedics 2012 Jun;35(6):e954-7.

30. Carvalho NN, Voss LA, Almeida MO, et al. Atypical femoral fractures during prolonged use of bisphosphonates: short-term responses to strontium ranelate and teriparatide. J Clin Endocrinol Metab 2011 Sep;96(9):267580.

31. Banffy MB, Vrahas MS, Ready JE. Nonoperative versus prophylactic treatment of bisphosphonate- associated femoral stress fractures. Clin Orthop Relat Res 2011 Jul;469(7):2028-34.

32. Ha YC, Cho MR, Park KH, et al. Is surgery necessary for femoral insufficiency fractures after long-term bisphosphonate therapy? Clin Orthop Relat Res 2010 Dec;468(12):3393-8.

33. Abrahamsen B, Eiken P, Eastell R. Subtrochanteric and diaphyseal femur fractures in patients treated with alendronate: a register-based national cohort study. J Bone Miner Res 2009 Jun;24(6):1095-102.

34. European Medicines Agency concludes class review of bisphosphonates and atypical fractures, EMA/CHMP/292784/2011, 2011 Apr.

35. Nieves JW, Cosman F. Atypical subtrochanteric and femoral shaft fractures and possible association with bisphosphonates. Curr Osteoporos Rep 2010 Mar;8(1):34-9.

36. Gunawardena I, Baxter M, Rasekh Y. Bisphosphonate-Related Subtrochanteric Femoral Fractures. Am J Geriatr Pharmacother 2011 Jun;9(3):194-8. 